\title{
Cardiac MRI in cardiomyopathies
}

\author{
Nishant Lahoti ${ }^{1}$, Richard J Jabbour ${ }^{2,3}$, Ben Ariff ${ }^{2,3}$ \& Brian Xiangzhi Wang *,2 iD \\ ${ }^{1}$ Barts \& The London School of Medicine \& Dentistry, Queen Mary University of London, London, UK \\ ${ }^{2}$ Department of Medicine, Faculty of Medicine, Imperial College London, London, UK \\ ${ }^{3}$ Imperial College Healthcare Trust, Hammersmith Hospital, London, UK \\ *Author for correspondence: Tel.: +44 792006 8530; brian.wang15@imperial.ac.uk
}

Heart failure affects $1-2 \%$ of the adult population and one of the main contributors to its development is cardiomyopathy. Assessing a patient's risk for adverse events in heart failure is challenging and made more difficult by the heterogenous phenotypic expression of the disease. Cardiac MRI has long been a gold standard measure of myocardial function and anatomy due to its high spatial and temporal resolution. More recently, it has been posited to play a more critical role in the diagnosis and prognosis of cardiomyopathy-related heart failure. Given the limitations of more commonly used imaging modalities, increasing the clinical use of cardiac magnetic resonance imaging could potentially improve the prognosis of specific subgroups of patients at risk of adverse cardiac events.

Lay abstract: Heart failure is a condition where the heart is unable to pump out enough blood to meet the body's daily needs. It can affect up to $2 \%$ of the adult population. One of the causes of heart failure is an intrinsic disease of the heart muscle, called cardiomyopathy. Assessing a patient's risk for events such as hospitalization and death in heart failure is challenging, and made more difficult by the wide variety of ways a patient can present with heart failure clinically. Cardiac MRI has long been a highly regarded imaging technique for heart function and shape due to the high level of detail it can show. More recently, it has been thought to play a more important role in investigating and predicting the course of cardiomyopathy-related heart failure. Given the limitations of more commonly used measurement techniques, increasing the clinical use of cardiac magnetic resonance imaging would potentially improve the outcomes and quality of life for patients suffering with cardiomyopathy.

First draft submitted: 21 December 2020; Accepted for publication: 22 March 2021; Published online: 22 April 2021

Keywords: cardiology $\bullet$ CMR $\bullet$ dilated cardiomyopathy $\bullet$ heart failure $\bullet$ hypertrophic cardiomyopathy

Heart failure due to cardiomyopathies is a significant problem, with conditions like dilated cardiomyopathy and hypertrophic cardiomyopathy exhibiting a prevalence of 1 per 500 in the general adult population [1]. Cardiomyopathic heart failure can be classified into two separate categories, based broadly on etiology: ischemic and nonischemic cardiomyopathies (NICMs). Ischemic cardiomyopathy is caused by coronary artery disease, whereas in NICMs and specifically dilated cardiomyopathy (DCM), the pathology has occurred in the absence of any ischemic and structural heart disease [1]. These categories present with the same constellation of symptoms (e.g., dyspnea, exercise intolerance, peripheral venous congestion), but the variations in pathophysiology mean that the use of cardiac MRI (CMR) in each of these categories is different. This review will primarily focus on NICMs. These can be further split into two main conditions, DCM and hypertrophic cardiomyopathy (HCM). HCM can cause heart failure with preserved ejection fraction, whereas DCM typically presents with heart failure with a reduced ejection fraction.

\section{DCM}

Dilated cardiomyopathy is a disease of heart muscle characterized by pathological dilatation of the ventricles in the absence of ischemic and structural heart disease, leading to impaired contraction and reduced cardiac output, resulting in the clinical syndrome of heart failure (HF) [1]. Its etiology is widespread, and some of the notable causes

Future $\because \cdots$ Medicine 


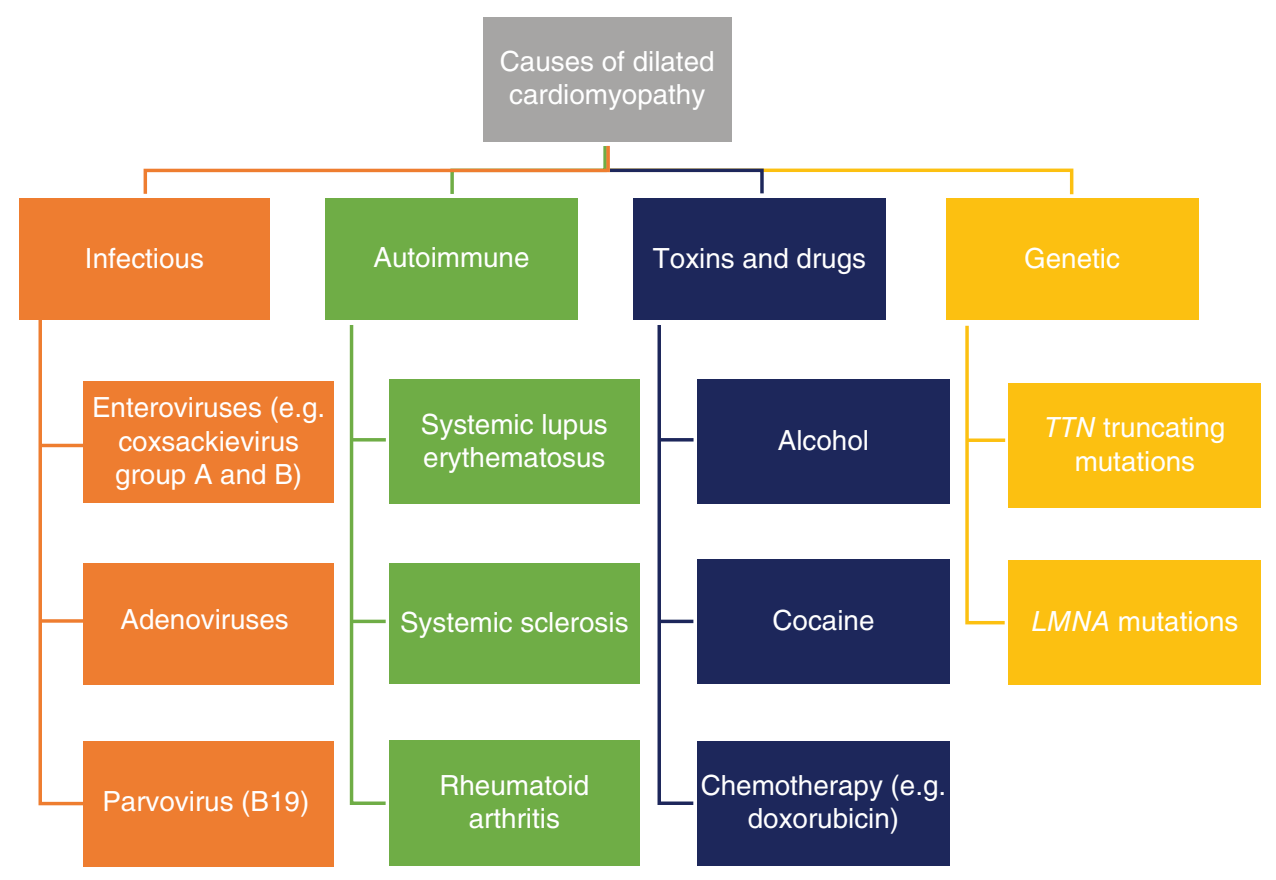

Figure 1. Flow diagram illustrating notable causative factors of dilated cardiomyopathy.

have been broadly summarized in Figure $1[2,3]$. There have been improvements in morbidity and mortality due to the introduction of pharmacological therapies such as angiotensin-converting enzyme inhibitors and $\beta$-blockers, and surgical therapies such as heart transplantation and implantable cardiac defibrillators (ICDs). However, there is room for improved diagnostic and prognostic techniques to more clearly delineate patients within what is a very heterogenous group in terms of etiology and disease progression [4]. This increasing 'personalization' of medicine could further improve outcomes in these patients through more accurate treatment guidelines and reliable markers and predictors of disease progression [5].

\section{$\mathrm{HCM}$}

HCM is defined as abnormal LV thickening without chamber dilation that is usually asymmetrical in the absence of an identifiable cause (e.g., hypertension) [6]. It is one of the most common genetic cardiomyopathies with a prevalence of approximately one in 500 adults. It is also etiologically variable, with at least 11 different genes that code for the cardiac sarcomere being implicated [7-9]. This variation translates to heterogeneity in phenotypic expression, with a wide spectrum of presentations, from asymptomatic to arrhythmias, advanced heart failure and sudden cardiac death [10]. As such, early HCM diagnosis is rare, with the majority of patients initially diagnosed for hypertension or Type II diabetes, leaving room for a more accurate and reliable noninvasive diagnostic method which would improve overall outcomes [11]. A key part of the definition of HCM is the presence of at least one thickened segment in a nondilated ventricle [12]. However, the location of these segments can vary, and the radiographic limitations of usual diagnostic methods such as transthoracic echocardiography mean there is a role for CMR to more accurately image these segments and identify a previously undiagnosed subset of HCM patients. Figure 2 more clearly illustrates the difference between HCM and DCM.

\section{Cardiac magnetic resonance imaging}

CMR has long been a gold standard noninvasive imaging technique for measurements of ventricular volume, mass and ejection fraction, all of which are critical factors in the diagnosis and prognosis of heart failure, due to its accuracy and reliability [13] and the use of key new imaging methods such as late gadolinium enhancement (LGE), T1 and T2 mapping. CMR images are three dimensional with a high spatial and temporal resolution in any plane and without ionizing radiation, making it well positioned as the imaging technique of choice for either diagnosis or repeated imaging for prognostic scanning [13]. Figure 3 illustrates a CMR image of a heart with HCM and a heart with DCM, along with key common findings. 


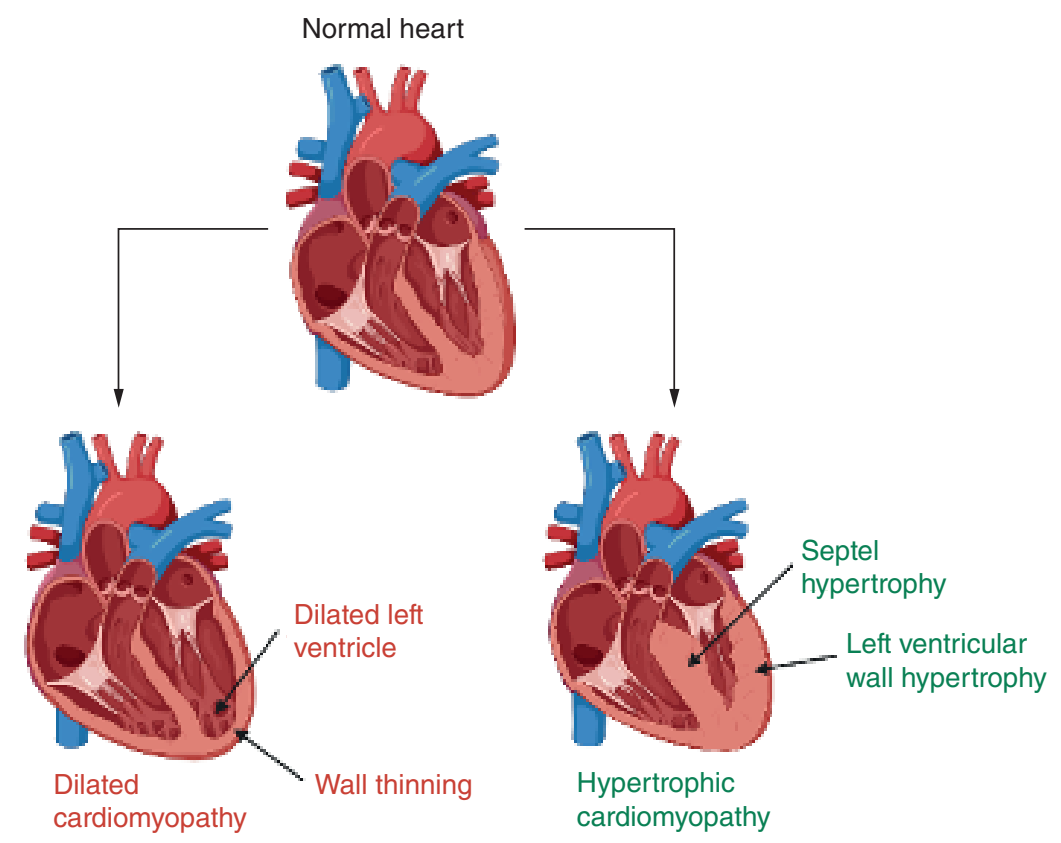

Figure 2. Difference in cardiac pathology between hypertrophic cardiomyopathy and dilated cardiomyopathy. Created with Biorender.com.

Figure 3. Hypertrophic versus dilated cardiomyopathy. Left: two-chamber delayed gadolinium enhancement imaging showing apical hypertrophic cardiomyopathy with diffuse mid-wall fibrosis in the hypertrophied segments. Right: Four-chamber SSFP imaging showing the typical appearance of dilated cardiomyopathy-related ventricular thinning.
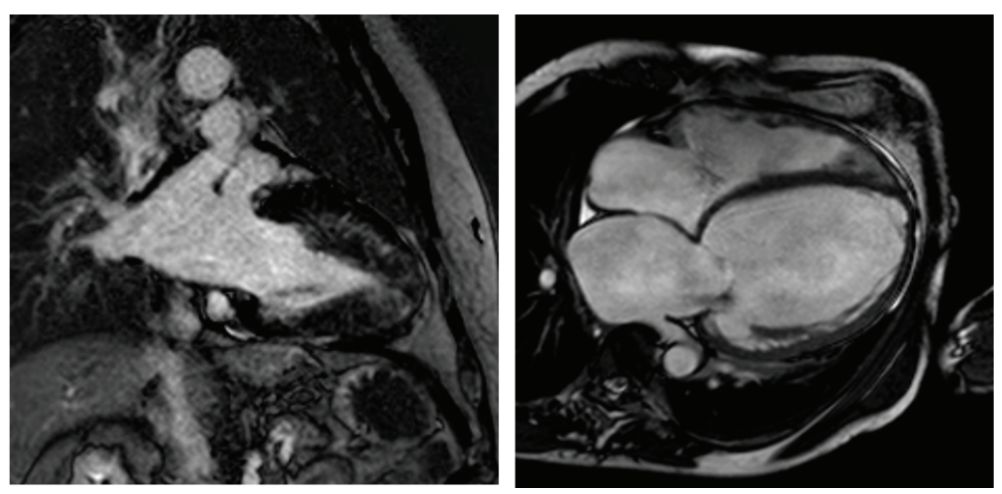

\section{Uses of CMR in DCM}

Measurement of ejection fraction for ICD implantation in DCM

ICD therapy is a mainstay in the prevention of sudden cardiac death (SCD) and reduction of overall mortality in DCM patients with reduced left ventricular ejection fraction [14-16]. The primary criterion for selection of candidates for primary prevention ICD is LVEF $<35 \%$ [17]. The imaging technique most commonly used to ascertain ejection fraction is transthoracic echocardiography (TTE), despite limitations such as high inter-observer variability and poorer resolution. CMR is considered more accurate and is often set as the gold standard for noninvasive LVEF assessment [18,19]. One of the advantages of further incorporation of CMR into the management of cardiomyopathies such as DCM is that the more accurate assessment of ejection fraction will hopefully lead to better risk stratification and more effective selection of patients for ICD implantation.

This is reinforced by work by Champ-Rigot et al. that investigated the discrepancy in ejection fraction measurements between CMR and TTE, and the impact this discrepancy has on clinical outcomes post-ICD implantation [20]. They retrospectively identified 173 ICM and NICM patients referred for primary prevention ICD implantation who had undergone both TTE and CMR less than 3 months prior to ICD implantation. Within the study population, it was shown that average LVEF measured by CMR (CMR-LVEF) (24\%) was significantly lower than the mean LVEF as measured by TTE (TTE-LVEF) (28\%), and overall, there was a mean difference between TTE and CMR of $4 \%$. Patients who reached the primary end point of a composite of death or appropriate 
device therapy (i.e., an episode of anti-tachycardia pacing and/or internal shocks delivered to terminate ventricular arrhythmia) could be differentiated by average CMR-LVEF but not by mean TTE-LVEF. These findings are further reinforced by the statistical analysis that showed CMR-LVEF $<22 \%$ was associated with an approximately $\sim$ twofold greater risk of the primary composite outcome, compared with $\sim 1$.6-fold in the equivalent (i.e., accounting for bias) TTE measurement of LVEF $<26 \%$. Among the 120 ICD-implanted ICM patients, the mean CMR-LVEF was found to be significantly lower among those who met the primary outcome than those who did not, but no such association was found with ICD-implanted NICM patients, in either CMR or TTE measurements. The study also examined the relevance of the presence of LGE, a sign of focal myocardial scarring. LGE was found in $76.9 \%$ patients, with the vast majority of those with scarring being ICM patients. LGE presence was found to be significantly associated with the primary composite of death and ADT, both by itself and in combination with CMR-LVEF measurements.

The overall survival was higher among patients with CMR-LVEF $\geq 22 \%$ than among patients with CMRLVEF $<22 \%$, whereas no such association was found with TTE-LVEF measurements. Overall, CMR and TTE did not correlate as well as expected, and exhibited markedly different outcome associations within a reasonably homogenous treatment group, with CMR-LVEF being more predictive of mortality. Furthermore, the presence of LGE proved to have predictive prognostic value, with the combination of CMR-LVEF $<22 \%$ and the presence of LGE being the most closely associated with the primary outcome [21]. The key outcome from this study is that given the demonstrated systemic overestimation of $2 \mathrm{D}$ echo, the guidelines around ICD implantation need to be reassessed. Given that the $35 \%$ cut-off value is not dependent on imaging technique, the choice of imaging technique could result in the reclassification of a significant number of patients for life-saving treatment. This is emphasized by other studies having shown that a TTE-LVEF within 5\% of guideline thresholds was predictive of reclassification by CMR for ICD implantation within those same guidelines [22,23]. One study showed that if the guidelines were adhered to using CMR-LVEF measurements as a prognostic guide, 19\% of those implanted patients would not have been treated based on their 2D-echo measurements, and that the patient cohort treated according to CMR-LVEF $<35 \%$ had reduced ADT rates compared with TTE-LVEF $<35 \%$ [24]. What is clear is that there is evidence within the literature that warrants reassessment of the guidelines, with indications that threshold values measured by CMR would be more effective in guiding treatment. Given the technical difficulties of accessing CMR on a wide scale, it would be impractical to restrict the guidelines to purely CMR measurements, but it might be worth framing a new TTE threshold in the light of a new CMR-based gold standard to mitigate for any systemic overestimation. Furthermore, increasing consideration needs to be given to the differing predictive values of LVEF in different cardiomyopathies. Unlike ICM patients, NICM patients who experienced ADT, survived cardiac arrest or sudden cardiac death could not be stratified according to CMR-LVEF [25]. A reduction in mortality with primary ICD implantation only in NICM patients presenting with an LV scar has been reported, and warrants further investigation [26]. The presence of LV scarring can be found using a CMR technique that uses LGE to highlight such areas.

\section{Late gadolinium enhancement in DCM}

LGE is a phenomenon seen on cardiac MRI where contrast and image delay are used together to highlight areas of scarring. It works on the basis that the contrast agent gadolinium accumulates only in the extracellular space, not within cells [27]. As such, it will accumulate more and take longer to dissipate in areas of increased extracellular volume, in other words, areas of fibrosis, than in healthy myocardium. If an image is taken with a delay compared with when the contrast is administered (i.e., late), then the gadolinium contrast agent will highlight areas of fibrosis where it has taken longer to dissipate from, in contrast to areas of healthy myocardium. LGE is a relatively common phenomenon in DCM patients, occurring in approximately $30 \%$ of patients and has been shown to have predictive value in prognostic outcomes such as all-cause mortality and sudden cardiac death [28,29]. Commonly, LGE in DCM presents in a linear pattern in the septal mid-wall, as illustrated in Figure 4.

However, multiple presentations of LGE have been identified in DCM patients, and examining the prognostic value of different extents, locations, or patterns could allow for optimization of the risk stratification value of this technique. Halliday et al. examined these different factors in 874 nonischemic DCM patients against a primary outcome of all-cause mortality, and a secondary outcome of a composite of SCD and aborted SCD [30]. As per previous literature, LGE was present in 34.3\% of the patient cohort, and this LGE cohort represented the more severely affected patients within the cohort, with lower LVEF and worse NYHA class. The most common location of LGE was: 


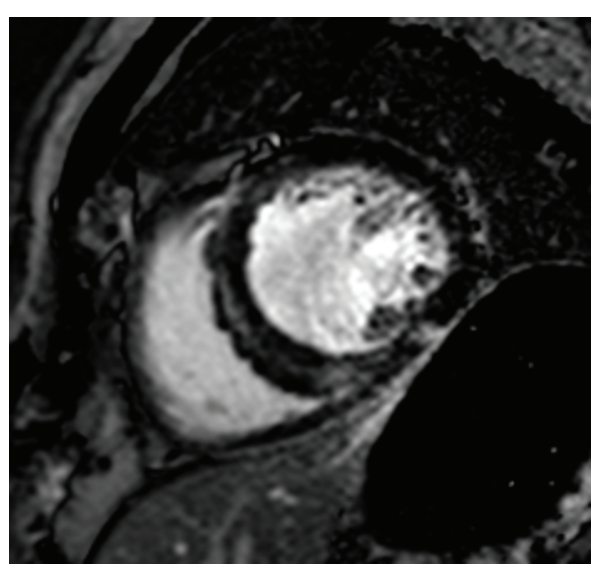

Figure 4. Delayed enhancement image demonstrating mid-wall fibrosis in a dilated cardiomyopathy.

\begin{tabular}{|c|c|c|}
\hline \multirow[t]{2}{*}{ LGE } & \multicolumn{2}{|c|}{ Adjusted hazard ratio } \\
\hline & All-cause mortality & $S C D+$ aborted $S C D$ \\
\hline \multicolumn{3}{|c|}{ Extent of LGE as a percentage of total myocardial mass: } \\
\hline $0-2.55 \%$ & 1.59 & 2.79 \\
\hline $2.55-5.10 \%$ & 1.56 & 3.86 \\
\hline$>5.10 \%$ & 2.31 & 4.87 \\
\hline \multicolumn{3}{|l|}{ Pattern of LGE: } \\
\hline Linear mid-wall & 1.70 & 3.21 \\
\hline Sub-epicardial & 1.29 & 5.54 \\
\hline Focal & 2.85 & 3.16 \\
\hline Multiple patterns of enhancement & 2.00 & 5.72 \\
\hline \multicolumn{3}{|l|}{ Location of LGE: } \\
\hline Septum only & 1.96 & 3.13 \\
\hline LV free-wall only & 0.77 & - \\
\hline Septum + LV free-wall & 1.99 & 5.82 \\
\hline
\end{tabular}

- Septum (16.2\%).

- Both the septum and the free wall of the left ventricle (13.3\%).

- Left ventricle free wall alone (4.8\%).

The most common patterns of LGE were:

- Linear mid-wall (21.1\%).

- Multiple patterns $(7.8 \%)$.

- Sub-epicardial (2.9\%).

- Focal (2.5\%).

The study's results are depicted in Table 1 [30]. Overall, as with previous studies in the literature, the presence of LGE was associated with increases in both the primary and secondary outcome [31]. Overall analysis showed that in all measured outcomes, location of LGE was more predictive than either extent or pattern of LGE. Septal LGE was the most predictive factor for the primary outcome, whereas for the secondary outcome, concomitant LGE in both the septum and free wall was more important, although LGE only in the septum did have some predictive value.

This can help in differentiating different etiological subsets of DCM. Idiopathic DCM is most commonly associated with septal mid-wall LGE whereas DCM associated with previous myocarditis is often associated with 
free-wall LGE [32]. Furthermore, genetic causes of DCM, for example, laminopathies, are characterized by mid-wall and subepicardial LGE patterns in multiple locations and have been associated with an overall worse prognosis than DCM associated with other causes [33]. Overall, it is clear that integrating CMR and LGE assessment into a model for predicting risk and guiding treatment such as ICD implantation in DCM patients would be of clinical benefit. Another interesting finding that would benefit from further work was the nonlinear relationship between LGE extent and outcome. If the relationship was modelled linearly, the study showed underestimation of risk in most patients while overestimating risk in patients with the largest extent. This indicates there is something deeper at play in terms of the pathophysiological effects of fibrosis, where the histological makeup of the scar might be more involved than previously thought.

There remain some issues with LGE as an imaging technique, as highlighted by Ehara and colleagues [34]. They highlighted the 'grey zone' of discrepant LGE assessment within what is normally a binary scale, with LGE either being present or not. They carried out a single-center, prospective, observational study in which 65 DCM patients who had had CMR were assessed by two different cardiologists and split into three groups:

1. Negative LGE (i.e., both cardiologists agreed no LGE was present, $n=29$ ).

2. Positive LGE (both cardiologists agreed that LGE was present, $n=20$ ).

3. Discrepant LGE (the two cardiologists disagreed on the presence of LGE, $n=16$ ).

These three groups were then followed up to assess the presence of early and late left ventricular reverse remodeling (LVRR), as well to measure any incidence of the composite end point of cardiovascular death, decompensated heart failure hospitalization, ventricular tachycardia and ICD discharge. The demographics across the three groups showed no significant differences in echocardiographic measurements such as EF or left ventricular end-diastolic diameter, or risk factors like age or serum levels of brain natriuretic peptide. Over the course of the follow-up period, the discrepant LGE group appeared to take up an intermediate position between negative LGE and positive LGE in terms of end points. With regards to changes in left ventricular end-diastolic diameter, there was no difference in between the individual groups. However, the overall trend was significant, with negative LGE showing the greatest percentage change, followed by the discrepant-LGE group and then the positive-LGE group. This was similarly reflected in changes in ejection fraction, where there were statistically significant differences between all three groups, as well as the same significant overall trend. In contrast, negative-LGE showed the greatest improvement in ejection fraction, positive-LGE the least, and discrepant-LGE maintaining an intermediate position inbetween the two. Overall, these changes suggest that changes in the shape and function of the heart, a prognostically positive sign, are related to the difference in LGE measurements that define the three groups. This is confirmed when assessing the frequency of early and late LVRR, which was shown to be significantly different between the three groups, with the negative-LGE group exhibiting the greatest amount of remodeling, positive-LGE the least, and discrepant-LGE inbetween the two.

What can be deduced from these results is that the discrepant-LGE group is at least prognostically distinct from the other two groups, indicating that the previous binary standard of visually assessing the presence of LGE might not be sufficient for the heterogenous DCM patient population. However, there are limitations to this study that need to be taken into account. For example, as with most radiological studies, there is potential for artifacts to influence findings even with all precautions being taken. Within the context of CMR, factors like poor breath-holding, and fluid (e.g., cerebrospinal fluid, pleural fluid in the form of an effusion) present in the imaging plane can all produce artifacts and are hard to control. Secondly, the patient population was very small, and so any broad conclusions must be tempered with the fact that these results need to be replicated in a larger, multi-center trial before the conclusions can be acted upon in terms of changing clinical practice. However, this study does provide the basis for larger investigation, given the possible clinical benefits of deferring any previously indicated ICD implantation. All three groups had relatively similar ejection fractions of $\sim 30 \%$ which would normally be an indication for defibrillator implantation for primary prevention. Given the increased propensity for both early and late LVRR, there is a basis for potentially deferring defibrillator implantation in patients who would fit into the negative or discrepant LGE groups, thus expanding CMR's prognostic role.

\section{Extracellular volume fraction measurements in DCM}

Issues surrounding the use of LGE include limitations in assessing other types of changes in heart muscle, such as diffuse myocardial fibrosis. Diffuse fibrosis is difficult to image via the LGE method, as there are fewer clearly 
defined boundaries between diffusely fibrotic areas and healthy myocardium than there are between focal scars and surrounding tissue. Thus, we need new techniques to image this type of scarring. Techniques, such as extracellular volume fraction (ECV) characterization via T1 mapping, have shown to be effective in imaging histologically validated diffuse fibrosis in NIDCM patients [35,36]. In a broader cardiac patient cohort, ECV has been shown to be correlated with adverse cardiac events, such as risk of death and hospitalization [37]. Changes in ECV are hypothesized to be reflective of changes in the interstitial space, and increased extracellular volume is associated with diffuse myocardial fibrosis [38]. Recently, it has been investigated to specifically examine its predictive value in NIDCM patients with comparison to LGE, where there remains an unmet need for accurate risk stratification [39]. The single-center study involved 240 NIDCM patients and assessed the correlation of their ECV overall, as well as correlation with specific cardio-anatomic locations, to a primary end point comprised of major adverse cardiac events (MACE), such as death from any cause or heart failure hospitalization. Within this cohort, with an average LVEF of $43 \%$, ECV was abnormal in at least 1 anatomical location in $58 \%$, compared with only $34 \%$ of the same cohort exhibiting nonischemic LGE. Over the average 4-year follow-up period, patients who met the primary end point had a higher mean ECV than those who did not. Furthermore, analysis showed the ability to stratify cardiac event-free survival as a function of increased ECV, such that a $10 \%$ increase in ECV showed 2.8-fold increase in risk of MACE. ECV also showed predictive value when combined with LVEF, as patients with LVEF $<30 \%$ showed a much more dramatic rise in rates of major adverse cardiac events (MACE) per year with increasing ECV tertile, compared with patients with LVEF $>30 \%$.

As well as the prognostic ability of overall ECV presence and extent, the high spatial resolution of CMR allows for more accurate localisation of specific areas of increased ECV within the heart. Compared with other cardioanatomic locations, ECV in the antero-septal area was most closely associated with MACE, but all areas had strong association with MACE. This fits with other studies that show scarring in the anteroseptal area is the most associated with recurrent ventricular tachycardia in patients with NIDCM [40]. Furthermore, there was a trend of increased MACE with the more areas that were affected, with annual MACE rates of $2.4 \%$ in patients with only one area of abnormal ECV, compared with 7.7.\% in patients with six abnormal areas of ECV. ECV maintained strong correlation with the outcome regardless of the presence of LGE, indicating relevancy in the stratification of a different patient group to LGE. While these results look promising, the strength of the conclusions is tempered somewhat by a few factors. It is hard to draw bold conclusions from the pure mortality data, as the sample size of patients that actually died is very small $(\mathrm{n}=14)$. This in part speaks to the patient cohort selected for, which overall was a representation of a relatively mild phenotypic expression of DCM. This study will need to be carried in a specific cohort of more unwell patients with more severe reduction in ejection fraction (EF) or ICD devices to make strong conclusions about ECV relating to mortality.

\section{Native T1 sequences in DCM}

While T1 mapping is critical for allowing calculations of ECV, native T1 imaging can also be used in early diagnosis of DCM. LV dilatation is typically the earliest noted hallmark on cardiac imaging of DCM. However, it can also develop as a normal physiological adaptive response to chronically sustained physical exertion, in a condition known as 'athlete's heart'. Endurance-focussed exercise typically can cause LV dilatation with significantly increased biventricular diameters and volumes, and consequently a mildly reduced LVEF (typically 45-55\%) [41]. In addition, some of these older patients with athlete's heart also have LGE present, thus creating further diagnostic uncertainty, and ensuring correct early diagnosis of cardiomyopathy is of vital importance for future prognosis and management [42]. A study by Mordi et al. showed the value of T1 mapping in this regard [43]. They investigated the differences in T1 mapping, T2 mapping and ECV measurements in 58 middle-aged males (21 healthy controls, 21 males with a history of aerobic exercise and LVEF $45-55 \%$ and 16 patients with DCM and LVEF 45-55\%). They showed that, while there were differences between the three groups in all the different imaging sequences, T1 mapping was the only independent discriminator between athletes and DCM patients. Going forward, this discriminative factor could prove critical in avoiding the consequences of an incorrect diagnosis - unnecessary exclusion from sport, or more importantly, SCD if undertaking exercise. Even with the limitations of the single center nature of this study and its small cohort, it provides the basis for further investigation of the utility of CMR in early DCM diagnosis to unearth a previously misdiagnosed subset of patients. 
Table 2. Summary of the various roles of cardiac MRI in dilated cardiomyopathy.

\begin{tabular}{|c|c|}
\hline CMR sequence & Relevant findings in DCM \\
\hline Ejection fraction measurement & $\begin{array}{l}\text { - A mean difference in EF measurement between TTE and CMR of } 4 \% \\
\text { - LVEF as measured by CMR being more predictive of mortality } \\
\text { - The choice of imaging technique for EF could result in the reclassification of a significant number of patients for life-saving } \\
\text { ICD implantation }\end{array}$ \\
\hline Late gadolinium enhancement & $\begin{array}{l}\text { - LGE occurs in approximately } 30 \% \text { of patients } \\
\text { - Different patterns and locations of LGE are associated with both different risks of adverse events and different etiological } \\
\text { subsets of DCM } \\
\text { - Not a purely binary measurement as variances in intensity of LGE can be predictive of variances in outcome }\end{array}$ \\
\hline Extracellular volume fraction & $\begin{array}{l}\text { - More accurate than LGE in imaging diffuse fibrosis, with increased predictive value for major adverse events, such as death } \\
\text { or hospitalization } \\
\text { - Increased percentage of ECV correlated with increased risk of adverse events } \\
\text { - Similar to LGE, the location of ECV had a predictive role } \\
\text { - Can be combined with LVEF to increase predictive value }\end{array}$ \\
\hline $\mathrm{T} 1$ sequences & $\begin{array}{l}\text { - T1 mapping shown to be most predictive factor in differentiating cause of initial LV dilatation, i.e., athlete's heart or the } \\
\text { initial stages of DCM in comparison to T2 mapping or ECV measurements } \\
\text { - Can be used in other organ systems, such as the liver, to track development of DCM to heart failure }\end{array}$ \\
\hline CMR: Cardiac MRI; DCM; Dilatec & $\begin{array}{l}\text { athy; ECV: Extracellular volume fraction; EF: Ejection fraction; ICD: Implanted cardioverter defibrillator; LGE: Late gadolinium enhancement; } \\
\text { on fraction; TTE; Transthoracic echocardiography. }\end{array}$ \\
\hline
\end{tabular}

\section{Future perspectives for CMR in DCM}

In broader terms, there is enough evidence to suggest that patients may benefit from increasing use of CMR in assessing risk and guiding treatment in HFrEF-related cardiomyopathies. As such, it could be beneficial to build a more extensive and multi-factorial risk model that would integrate multiple predictive factors, both clinical, such as NYHA class, and radiological, such as CMR-LVEF, presence of LGE, and extent and location of ECV that might provide a more accurate answer as to which $\mathrm{HFrEF}$ patient subgroups should receive what treatment.

An interesting emerging use of CMR in DCM is that of measuring liver T1 relaxation times as a proxy for measuring hepatic congestion and oedema, a classic sequelae of heart failure. Huber $e t$ al. hypothesized that patients with IDCM with acute HF may have increased hepatic native TI relaxation times in liver parenchyma due to hepatic congestion compared with DCM patients that had not progressed to heart failure [44]. Furthermore, they analysed whether this potential change in hepatic relaxation time would be predictive of adverse events such as hospitalization, both as a standalone measure and in comparison, to other predictive values, such as LVEF. This retrospective cross-sectional study examined 55 patients (20 healthy control subjects and 35 consecutively registered patients with IDCM). Within this small sample size, they found that native T1 relaxation time of the liver was a clearer differentiator of DCM patients with or without heart failure than LVEF and LV strain. LVEF decreased continuously with disease severity (from healthy control subjects to DCM without and DCM with HF), but hepatic native $\mathrm{T} 1$ relaxation time only significantly. Furthermore, on a technical level, the hepatic relaxation times were measures on standard short-axis cardiac MRI T1-weighted maps, minimising a technical hurdle to introducing this into clinical practice. These findings, albeit limited by a small sample size, are a good illustration of how one can utilise the multiparametric nature of CMR going forward and open up a range of diagnostic measures to more accurately and successfully manage DCM and its complications. CMR can be used both in the initial diagnostic phase for DCM, as well as charting its progression and complications in multiple organs while predicting prognosis throughout. A summary of the role of CMR in DCM can be found in Table 2.

\section{Uses of CMR in HCM}

Within HFpEF, it is important to be able to differentiate the causes of left ventricular hypertrophy (LVH) to enable correct management. With regards to differentiating cardiomyopathies, it is important to be able to isolate causes such as hypertrophic cardiomyopathy (HCM) from hypertension-related remodeling. CMR is regarded as the gold standard in assessing $\mathrm{LVH}$, and recent advancements in techniques such as T1 and T2 mapping and ECV measurements have further highlighted the critical role it can play $[18,45]$.

\section{Diagnostic potential of CMR in HCM}

The diagnostic potential of CMR in differential diagnosis in HCM was highlighted by an investigation into the value of systematic CMR on 154 nonischemic HFpEF patients [11]. CMR unearthed previously unknown diagnoses in $27 \%$ of the cohort ( 42 patients). The new diagnoses broke down as follows: epicardial coronary artery disease 

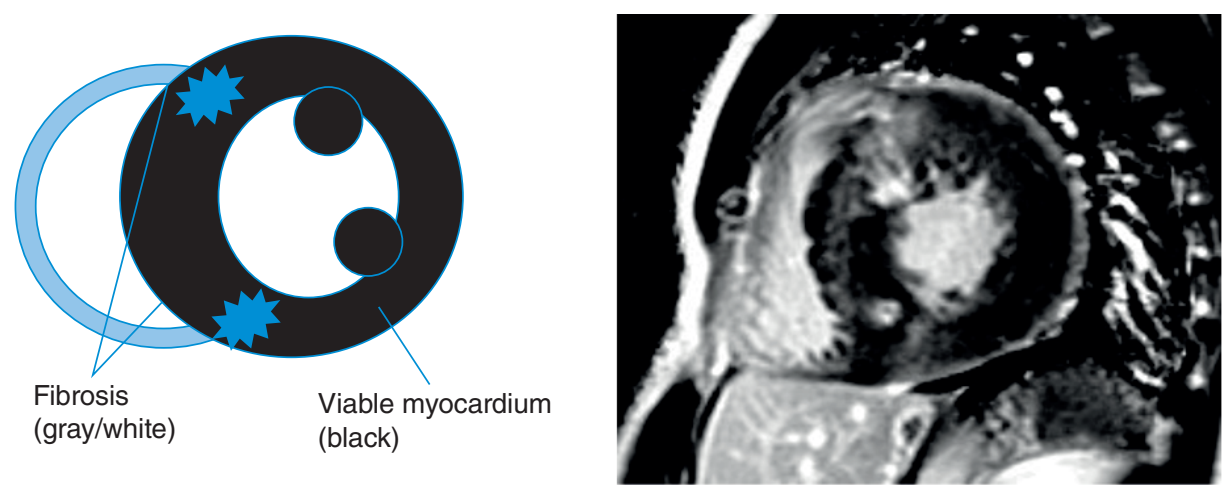

Figure 5. An illustration of ventricular wall thickening and areas of fibrosis in a heart with hypertrophic cardiomyopathy.

based on MI or ischemia $(n=20)$, microvascular dysfunction $(n=11), \operatorname{HCM}(n=10)$ and constrictive pericarditis $(\mathrm{n}=5)$, with some patients finding multiple new diagnoses. Furthermore, between the new diagnoses group, and the rest of the cohort, there was no other significant difference, either demographically or clinically, that would predict the presence of an undiagnosed condition. Analysis showed lower event-free rates in the new-diagnosis group, and new diagnosis on CMR was shown to be predictive of the primary end point of death and heart failure hospitalization. With specific regard to HCM, TTE measurements carried out on the same group showed a systematic underestimation of wall thickness and could only diagnose half of the new cases that were diagnosed by CMR. This study highlights some of the issues in diagnosing HCM in normal clinical practice, especially when comparing left ventricular hypertrophy (LVH) in HCM patients versus hypertensive patients. Hypertensive LVH usually presents concentrically with a typical maximum wall thickness of $\sim 16 \mathrm{~mm}$, whereas HCM-related hypertrophy is usually a lot more asymmetrical and of variable thickness [46,47]. Differentiating between the two with an imaging methodology as subjective and limited as TTE is very difficult and speaks to the large gap in rates of diagnosis seen in this study, and the need for imaging techniques with much higher resolution and delineation capacity, such as CMR [48,49].

\section{Role of late gadolinium enhancement in HCM}

The histological presence of myocardial fibrosis is a key causative factor in the pathophysiology of HCM. Figure 5 highlights how patchy fibrosis appears on a CMR image of HCM. The fibrotic tissue is naturally stiffer than the muscle it replaced, hence the increased LV stiffness and resultant diastolic dysfunction [8]. However, the association between LGE and HCM is not clear cut. The literature has not produced a clear picture. Early work showed a correlation between LGE extent and worse outcomes, such as sudden cardiac death [50,51] but recent larger studies have cast doubt on these findings. One such study included $711 \mathrm{HCM}$ patients followed over the course of approximately 3.5 years. This study identified LGE in $66 \%$ of the patient cohort, but after adjustment for ejection fraction, this was not independently associated with any worse outcome [52]. A larger study of 1293 HCM patients did find some association between the presence of LGE and risk of sudden death events, but there were significant limitations in that the sample size of patients meeting the outcomes was small and after adjustment for typical risk factors, the association was somewhat weakened [53,54].

However, a recent meta-analyses of seven studies encompassing $\sim 3000$ patients showed strong association between LGE and SCD [55]. Additionally, percentage of LGE as measured on CMR was shown to be more predictive of cardiac events such as SCD, aborted SCD, sustained ventricular tachycardia, or appropriate ICD discharge than the two clinical risk stratification tools currently being used [56].

\section{T1 mapping \& extracellular volume fraction measurements in HCM}

However, a more clinically beneficial area of interest might be the diffuse fibrosis that precedes the more focal fibrotic stage of HCM. Multiple endomyocardial biopsy and autopsy studies in HCM patients have demonstrated the presence of interstitial fibrosis, and the resultant increased ventricular stiffness is key to the cardiac dysfunction seen in this group [57,58]. Furthermore, focal fibrosis is more associated with a lack of LVRR, so the preceding stage might be more amenable to treatment. All of this points to the fact that a noninvasive method of consistently 
tracking diffuse fibrosis would be clinically beneficial. This is where the emerging technique of CMR T1 mapping, in other words, numerical quantification of $\mathrm{T} 1$ relaxation times, has the potential to be very important in the diagnosis and risk stratification of HCM. These numerical values can be color coded and allow for detailed myocardial maps with very high resolution to identify even relatively small variations of T1 times, allowing for visualization of interstitial fibrosis [38]. T1 imaging has been validated against histological findings in a wide spectrum conditions, including HCM [59]. These T1 maps can be used to calculate other very useful measurements, such as ECV [60]. In fact, compared with both native T1 and postcontrast T1, ECV has been shown to demonstrate the best correlation with diffuse myocardial fibrosis [59]. Su et al. showed that ECV was correlated with peak filling rates in the HFpEF patients but not in the hypertensive controls or the comparative HFrEF group, thus indicating a singular connection between CMR-quantified myocardial fibrosis and ventricular function specifically in HFpEF patients [61]. This was emphasized in a wider study of $117 \mathrm{HFpEF}$ patients where ECV correlated with clinical parameters, such as brain natriuretic peptide and 6-min walk distance, as well as cardiac events such as heart failure hospitalizations and cardiac deaths [62]. Recent work by Romell et al. compared $24 \mathrm{HFpEF}$ patients with 12 control patients, who presented for evaluation of atypical chest pain, accompanied by a substantial cardiovascular risk profile, but without heart failure symptoms [63]. Overall, ECV correlated highly with myocardial stiffness and the HFpEF patients showed elevated ECV compared with the control group. On further analysis, ECV remained the only independent predictor of myocardial stiffness, suggesting a diagnostic value of ECV in HFpEF independent of echocardiographic assessment.

However, there are still limitations to overcome. T1 mapping protocols are yet to be properly standardized, meaning comparisons between different centers are difficult. Furthermore, many of the trials only have small sample sizes and single center bias [60]. Overall T1 mapping has the potential to be an effective tool for diagnosis and risk stratification in HCM but requires testing in larger-scale multicenter trials with more standardized imaging parameters and techniques.

\section{T2 mapping in HCM}

The presence of hyperintense signals on T2-weighted CMR images has recently been noted in HCM patients, with myocardial high T2 signal being significantly predictive of life-threatening arrhythmia in HCM patients [64-66]. Such signals have highlighted the areas of ischemic infarction after an acute myocardial attack, and ischemia is a common finding in HCM, due to the imbalance of the hypertrophic myocardium and blood supply to tissue [67,68]. As such, high T2 signal has been hypothesized to be a marker of myocardial injury in HCM patients, meaning that T2-weighted CMR can provide both pathophysiological insights to the progression of HCM and form part of an integrated risk stratification model, to mitigate the effects of this myocardial injury in certain subgroups of patients that are more affected. This was the basis of a study carried out by Chen et al., who prospectively investigated the potential correlation between high-sensitivity cardiac troponin $\mathrm{T}$ (hs-c $\mathrm{TnT}$ ), a classical biomarker of myocardial injury noted to be elevated in some HCM patients, and the presence and extent of hyperintense signals on T2-weighted MRI [69]. In a patient cohort of 44, it was found that 33 patients had evidence of positive findings on T2 imaging. This T2 positive subgroup had significantly smaller LV cavities and higher cardiac troponin levels. This was reinforced by analysis that showed a statistically significant positively correlating trend of increased hs-cTnT levels with increasing extent of high T2 signal. Interestingly, neither positive findings on T2 imaging nor hs-cTnT were corelated with the extent of LGE, as well as mixed evidence in the literature correlating size, shape and location of LGE with the location of hyperintense T2 signals $[64,66]$. This might implicate a different pathophysiological process at work than the focal fibrotic scarring highlighted by LGE. Given the small sample size, and lack of cross-correlation with clinical outcomes, as well as the lack of truly quantitative evaluation of the T2 imaging, the conclusions that can be drawn are not strong. However, given the seemingly high prevalence of hyperintensity on T2 imaging within the cohort, it would be worth further investigation, correlating T2 imaging with more directly localising signs of myocardial injury.

The summary of the various roles of CMR in hypertrophic cardiomyopathy is presented in Table 3.

\section{Conclusion}

In summary, there are myriad diagnostic and prognostic uses of CMR in both DCM and HCM. In DCM, the diagnostic role of CMR in accurately differentiating between different early causes of LV dilatation, such as athlete's heart will become crucial, and that early diagnosis will allow for a reduction in the risk of disease progression and likelihood of hospitalization. CMR's prognostic role in evaluating pacemaker suitability will become invaluable, and 
Table 3. Summary of the various roles of cardiac magnetic resonance imaging in hypertrophic cardiomyopathy.

\begin{tabular}{|l|l|}
\hline CMR sequence & Relevant findings in HCM \\
\hline Diagnostic potential & - CMR can more easily differentiate different causes of LVH, i.e., HCM vs hypertensive cardiomyopathy \\
\hline LGE & - Lack of correlation between presence of LGE and outcomes in HCM \\
\hline T1 mapping and ECV measurements & $\begin{array}{l}\text { - Small scale studies have shown that ECV maps have predictive value for adverse outcomes } \\
\text { - Needs to be tested in larger, multi-center trials }\end{array}$ \\
\hline T2 mapping & $\begin{array}{l}\text { - T2-weighted CMR can provide both pathophysiological insights to the progression of HCM } \\
\text { - High intensity signals on T2-weighted images have been shown to correlate with troponin T levels and hypothesized to be a } \\
\text { marker of myocardial injury in HCM patients }\end{array}$
\end{tabular}

CMR: Cardiac MRI; ECV: Extracellular volume fraction; HCM; Hypertrophic cardiomyopathy; LGE: Late gadolinium enhancement; LVH: Left ventricular hypertrophy.

will allow for increased accuracy of EF stratification, which in turn will significantly improve treatment outcomes. Furthermore, indicators, such as LGE presence and location and extracellular volume, will allow for much more targeted, individualized treatment plans, again leading to an overall improvement in patient outcome. Newly developed indicators, such as measuring hepatic congestion as a tracker for disease progression, would allow for the development of a wider set of pathological and radiological matrices to more accurately track all aspects of disease progression, enabling more holistic patient care. In HCM, CMR can have a similar differentiating diagnostic role in assessing between different causes of $\mathrm{LVH}$, such as hypertensive cardiomyopathy and allowing for early diagnosis and treatment to reduce overall risk of hospitalisation and death. Prognostically, the picture is less clear. The role of LGE in HCM is unclear, and is yet to be proven as a prognostic indicator in this disease. However, other aspects of the underlying pathophysiology of HCM have been illuminated by CMR, such as the high-intensity signals on T2-weighted images, which have been shown to correlate with troponin $\mathrm{T}$ levels, potentially indicating a underlying role for myocardial ischaemia in the progression of HCM.

\section{Future perspective}

There remains numerous issues with CMR as a technique, to investigate cardiomyopathies in general, which make assessing progress in this field difficult. For example, there are barriers to wider clinical use due to practicality and availability, especially with the more widely available, cheaper and bedside nature of the more commonly used 2Decho imaging technique. One can therefore imagine the course of this field over the next 5-10 years being directed to overcoming these barriers. Clearer definitions on the conditions being studied, as well as more consistency in imaging protocols, to facilitate comparing studies involving CMR and the various nonischemic cardiomyopathies easier. Different studies working by different definitions for nonischemic cardiomyopathy compared with dilated cardiomyopathy compared with ischemic cardiomyopathy can make it somewhat difficult to collate the literature and make definitive comparisons between these conditions. A universal standardized definition of diagnostic and exclusionary criteria for each condition, that could be partly based on and incorporate CMR findings, would be a particularly useful goal for research going forward.

Overall, CMR is proving a very promising imaging modality to aid in the diagnosis and prognosis in some very heterogenous patient groups, and warrants further investigation to inform updated treatment guidelines in these conditions.

\section{Financial \& competing interests disclosure}

The authors have no relevant affiliations or financial involvement with any organization or entity with a financial interest in or financial conflict with the subject matter or materials discussed in the manuscript. This includes employment, consultancies, honoraria, stock ownership or options, expert testimony, grants or patents received or pending, or royalties.

No writing assistance was utilized in the production of this manuscript.

\section{Open access}

This work is licensed under the Creative Commons Attribution 4.0 License. To view a copy of this license, visit http://creativecommons.org/licenses/by/4.0/ 
Executive summary

\section{Dilated cardiomyopathy}

- A disease of heart muscle characterized by pathological dilatation of the ventricles in the heart in the absence of ischemic and structural heart disease, leading to impaired contraction and reduced cardiac output.

Hypertrophic cardiomyopathy

- Abnormal asymmetrical LV thickening without chamber dilation in the absence of an identifiable cause.

Cardiac MRI

- A gold standard noninvasive imaging technique for measurements of ventricular volume, mass, and ejection fraction due to high spatial and temporal resolution.

Uses of CMR in dilated cardiomyopathy

Measurement of ejection fraction for ICD implantation in DCM

- A mean difference in ejection fraction (EF) measurement between transthoracic echocardiography and cardiac magnetic resonance imaging (CMR) of $4 \%$.

- LVEF as measured by CMR being more predictive of mortality.

- The choice of imaging technique for EF could result in the reclassification of a significant number of patients for ICD implantation.

Late gadolinium enhancement in DCM

- LGE occurs in approximately $30 \%$ of patients.

- Different patterns and locations of LGE are associated with both different risks of adverse events and different etiologies of DCM.

- Variances in intensity of LGE can be predictive of variances in outcome.

Extracellular volume fraction in DCM

- More accurate than LGE in imaging diffuse fibrosis, with increased predictive value for death or hospitalization.

- Increased percentage and location of ECV correlated with increased risk of adverse events.

Native T1 imaging in DCM

- T1 mapping shown to be most predictive factor in differentiating cause of initial LV dilatation, in other words, athlete's heart or DCM.

- Can potentially be used in the liver to track development of DCM to heart failure.

Future perspective

- CMR can be used to build a more extensive and multi-factorial model that would integrate multiple predictive clinical and radiological factors to inform about risk and guide treatment.

Uses of CMR in hypertrophic cardiomyopathy

Diagnostic potential of CMR in HCM

- CMR can more easily differentiate different causes of LVH, in other words, HCM versus hypertensive cardiomyopathy.

Late gadolinium enhancement in HCM

- Lack of correlation between presence of LGE and outcomes in HCM.

- The literature has conflicting information, and the picture is not clear.

T1 mapping \& ECV in HCM

- Small scale studies have shown that ECV maps have predictive value for adverse outcomes.

- Needs to be tested in larger, multi-center trials.

T2 mapping in HCM

- High intensity signals on T2-weighted images have been shown to correlate with troponin T levels and hypothesized to be a marker of myocardial injury in HCM patients.

Future perspective

- Important to overcome barriers to wider clinical use due to practicality and availability, especially with the more widely available, cheaper and bedside nature of the more commonly used 2D-echo imaging technique.

- A goal of a universal standardized definition of diagnostic and exclusionary criteria for each condition, that could be partly based on and incorporate CMR findings.

\section{References}

Papers of special note have been highlighted as: $\bullet$ of interest; $\bullet \bullet$ of considerable interest

1. McKenna WJ, Maron BJ, Thiene G. Classification, epidemiology, and global burden of cardiomyopathies. Circ. Res. 121(7), 722-730 (2017).

2. Trachtenberg BH, Hare JM. Inflammatory cardiomyopathic syndromes. Circ. Res. 121(7), 803-818 (2017).

3. Schultheiss HP, Fairweather D, Caforio ALP et al. Dilated cardiomyopathy. Nat. Rev. Dis. Primers 5(1), 32 (2019). 
4. McNamara DM, Starling RC, Cooper LT et al. Clinical and demographic predictors of outcomes in recent onset dilated cardiomyopathy: results of the IMAC (Intervention in Myocarditis and Acute Cardiomyopathy)-2 study. J. Am. Coll. Cardiol. 58(11), 1112-1118 (2011).

5. Halliday BP, Cleland JGF, Goldberger JJ, Prasad SK. Personalizing risk stratification for sudden death in dilated cardiomyopathy: the past, present, and future. Circulation 136(2), 215-231 (2017).

6. Hensley N, Dietrich J, Nyhan D, Mitter N, Yee MS, Brady M. Hypertrophic cardiomyopathy: a review. Anesth. Analg. 120(3), 554-569 (2015).

7. Masarone D, Kaski JP, Pacileo G et al. Epidemiology and clinical aspects of genetic cardiomyopathies. Heart Fail. Clin. 14(2), 119-128 (2018).

8. Maron BJ, Ommen SR, Semsarian C, Spirito P, Olivotto I, Maron MS. Hypertrophic cardiomyopathy: present and future, with translation into contemporary cardiovascular medicine. J. Am. Coll. Cardiol. 64(1), 83-99 (2014).

9. Fourey D, Care M, Siminovitch KA et al. Prevalence and clinical implication of double mutations in hypertrophic cardiomyopathy: revisiting the gene-dose effect. Circ. Cardiovasc. Genet. 10(2), 1685 (2017).

10. Maron BJ. Clinical course and management of hypertrophic cardiomyopathy. N. Engl. J. Med. 379(7), 655-668 (2018).

11. Kanagala $P$, Cheng ASH, Singh A et al. Diagnostic and prognostic utility of cardiovascular magnetic resonance imaging in heart failure with preserved ejection fraction - implications for clinical trials. J. Cardiovasc. Magn. Reson. 20(1), 4 (2018).

- This study was notable for highlighting the diagnostic potential of cardiac MRI (CMR) for reclassifying hitherto undiagnosed patients with heart failure with preserved ejection fraction.

12. Brenes JC, Doltra A, Prat S. Cardiac magnetic resonance imaging in the evaluation of patients with hypertrophic cardiomyopathy. Glob. Cardiol. Sci. Pract. 2018(3), 22 (2018).

13. Hundley WG, Bluemke DA, Finn JP et al. ACCF/ACR/AHA/NASCI/SCMR 2010 expert consensus document on cardiovascular magnetic resonance: a report of the American College of Cardiology Foundation Task Force on Expert Consensus Documents. J. Am. Coll. Cardiol. 55(23), 2614-2662 (2010).

14. Connolly SJ, Hallstrom AP, Cappato R et al. Meta-analysis of the implantable cardioverter defibrillator secondary prevention trials. AVID, CASH and CIDS studies. Antiarrhythmics vs Implantable Defibrillator study. Cardiac Arrest Study Hamburg. Canadian Implantable Defibrillator Study. Eur. Heart J. 21(24), 2071-2078 (2000).

15. Desai AS, Fang JC, Maisel WH, Baughman KL. Implantable defibrillators for the prevention of mortality in patients with nonischemic cardiomyopathy: a meta-analysis of randomized controlled trials. JAMA 292(23), 2874-2879 (2004).

16. Stavrakis S, Asad Z, Reynolds D. Implantable cardioverter defibrillators for primary prevention of mortality in patients with nonischemic cardiomyopathy: a meta-analysis of randomized controlled trials. J. Cardiovasc. Electrophysiol. 28(6), 659-665 (2017).

17. Al-Khatib SM, Stevenson WG, Ackerman MJ et al. 2017 AHA/ACC/HRS guideline for management of patients with ventricular arrhythmias and the prevention of sudden cardiac death: executive summary: a report of the American College of Cardiology/American Heart Association Task Force on Clinical Practice Guidelines and the Heart Rhythm Society. Heart Rhythm 15(10), e190-e252 (2018).

18. Bellenger NG, Burgess MI, Ray SG et al. Comparison of left ventricular ejection fraction and volumes in heart failure by echocardiography, radionuclide ventriculography and cardiovascular magnetic resonance; are they interchangeable? Eur. Heart J. 21(16), 1387-1396 (2000).

19. McMurray JJ, Adamopoulos S, Anker SD et al. ESC guidelines for the diagnosis and treatment of acute and chronic heart failure 2012: The Task Force for the Diagnosis and Treatment of Acute and Chronic Heart Failure 2012 of the European Society of Cardiology. Developed in collaboration with the Heart Failure Association (HFA) of the ESC. Eur. J. Heart Fail. 14(8), 803-869 (2012).

20. Champ-Rigot L, Gay P, Seita F et al. Clinical outcomes after primary prevention defibrillator implantation are better predicted when the left ventricular ejection fraction is assessed by cardiovascular magnetic resonance. J. Cardiovasc. Magn Reson. 22(1), 48 (2020).

-. This study highlighted the key disparities in outcome when CMR is used to measure ejection fraction rather than transthoracic echocardiography.

21. Nazarian S, Bluemke DA, Lardo AC et al. Magnetic resonance assessment of the substrate for inducible ventricular tachycardia in nonischemic cardiomyopathy. Circulation 112(18), 2821-2825 (2005).

22. de Haan S, de Boer K, Commandeur J, Beek AM, van Rossum AC, Allaart CP. Assessment of left ventricular ejection fraction in patients eligible for ICD therapy: discrepancy between cardiac magnetic resonance imaging and 2D echocardiography. Neth. Heart J. 22(10), 449-455 (2014).

23. Rayatzadeh H, Patel SJ, Hauser TH et al. Volumetric left ventricular ejection fraction is superior to 2-dimensional echocardiography for risk stratification of patients for primary prevention implantable cardioverter-defibrillator implantation. Am. J. Cardiol. 111(8), 1175-1179 (2013).

24. Rijnierse MT, van der Lingen AL, Weiland MT et al. Clinical impact of cardiac magnetic resonance imaging versus echocardiography-guided patient selection for primary prevention implantable cardioverter defibrillator therapy. Am. J. Cardiol. 116(3), 406-412 (2015). 
25. Gao P, Yee R, Gula L et al. Prediction of arrhythmic events in ischemic and dilated cardiomyopathy patients referred for implantable cardiac defibrillator: evaluation of multiple scar quantification measures for late gadolinium enhancement magnetic resonance imaging. Circ. Cardiovasc. Imaging 5(4), 448-456 (2012).

26. Gutman SJ, Costello BT, Papapostolou $S$ et al. Reduction in mortality from implantable cardioverter-defibrillators in nonischemic cardiomyopathy patients is dependent on the presence of left ventricular scar. Eur. Heart J. 40(6), 542-550 (2019).

27. Kim RJ, Fieno DS, Parrish TB et al. Relationship of MRI delayed contrast enhancement to irreversible injury, infarct age, and contractile function. Circulation 100(19), 1992-2002 (1999).

28. Halliday BP, Gulati A, Ali A et al. Association between midwall late gadolinium enhancement and sudden cardiac death in patients with dilated cardiomyopathy and mild and moderate left ventricular systolic dysfunction. Circulation 135(22), 2106-2115 (2017).

29. Gulati A, Jabbour A, Ismail TF et al. Association of fibrosis with mortality and sudden cardiac death in patients with nonischemic dilated cardiomyopathy. JAMA 309(9), 896-908 (2013).

30. Halliday BP, Baksi AJ, Gulati A et al. Outcome in dilated cardiomyopathy related to the extent, location, and pattern of late gadolinium enhancement. JACC Cardiovasc. Imaging 12 (8 Pt 2), 1645-1655 (2019).

- This study highlighted notable associations between various characteristics of late gadolinium enhancement (LGE) and dilated cardiomyopathy outcome to illustrate that the mere presence of LGE was not the only predictive factor in prognosis.

31. Disertori M, Rigoni M, Pace $\mathrm{N}$ et al. Myocardial fibrosis assessment by LGE Is a powerful predictor of ventricular tachyarrhythmias in ischemic and nonischemic lv dysfunction: a meta-analysis. JACC Cardiovasc. Imaging 9(9), 1046-1055 (2016).

32. Mahrholdt H, Wagner A, Deluigi CC et al. Presentation, patterns of myocardial damage, and clinical course of viral myocarditis. Circulation 114(15), 1581-1590 (2006)

33. Hasselberg NE, Haland TF, Saberniak J et al. Lamin A/C cardiomyopathy: young onset, high penetrance, and frequent need for heart transplantation. Eur. Heart J. 39(10), 853-860 (2018)

34. Ehara S, Matsumoto K, Kitada R, Nishimura S, Shimada K, Yoshiyama M. Clinical significance of discrepant mid-wall late gadolinium enhancement in patients with nonischemic dilated cardiomyopathy. Heart Vessels 33(12), 1482-1489 (2018).

-. Shows the limitations and areas for improvement when using the presence of LGE to draw conclusions regarding prognosis and risk of adverse outcomes.

35. Kehr E, Sono M, Chugh SS, Jerosch-Herold M. Gadolinium-enhanced magnetic resonance imaging for detection and quantification of fibrosis in human myocardium in vitro. Int. J. Cardiovasc. Imaging 24(1), 61-68 (2008).

36. Coelho-Filho OR, Mongeon FP, Mitchell R et al. Role of transcytolemmal water-exchange in magnetic resonance measurements of diffuse myocardial fibrosis in hypertensive heart disease. Circ. Cardiovasc. Imaging 6(1), 134-141 (2013).

37. Wong TC, Piehler K, Meier CG et al. Association between extracellular matrix expansion quantified by cardiovascular magnetic resonance and short-term mortality. Circulation 126(10), 1206-1216 (2012).

38. Taylor AJ, Salerno M, Dharmakumar R, Jerosch-Herold M. T1 mapping: basic techniques and clinical applications. JACC Cardiovasc. Imaging 9(1), 67-81 (2016).

39. Vita T, Grani C, Abbasi SA et al. Comparing CMR mapping methods and myocardial patterns toward heart failure outcomes in nonischemic dilated cardiomyopathy. JACC Cardiovasc. Imaging 12(8 Pt 2), 1659-1669 (2019).

40. Oloriz T, Silberbauer J, Maccabelli G et al. Catheter ablation of ventricular arrhythmia in nonischemic cardiomyopathy: anteroseptal versus inferolateral scar sub-types. Circ. Arrhythm. Electrophysiol. 7(3), 414-423 (2014).

41. Zilinski JL, Contursi ME, Isaacs SK et al. Myocardial adaptations to recreational marathon training among middle-aged men. Circ. Cardiovasc. Imaging 8(2), e002487 (2015).

42. Galderisi M, Cardim N, D'Andrea A et al. The multi-modality cardiac imaging approach to the Athlete's heart: an expert consensus of the European Association of Cardiovascular Imaging. Eur. Heart J. Cardiovasc. Imaging 16(4), 353 (2015).

43. Mordi I, Carrick D, Bezerra H, Tzemos N. T1 and T2 mapping for early diagnosis of dilated nonischemic cardiomyopathy in middle-aged patients and differentiation from normal physiological adaptation. Eur. Heart J. Cardiovasc. Imaging 17(7), 797-803 (2016).

-. This study highlighted the specific role of T1 mapping in differentiating the early stages of dilated cardiomyopathy from other conditions, such as athlete's heart, that would present similarly on more commonly used imaging techniques.

44. Huber AT, Razakamanantsoa L, Lamy J et al. Multiparametric differentiation of idiopathic dilated cardiomyopathy with and without congestive heart failure by means of cardiac and hepatic T1-weighted MRI mapping. AJR Am. J. Roentgenol. 215(1), $79-86$ (2020).

- This study highlighted an interesting extra-cardiac use of CMR to monitor dilated cardiomyopathy progression.

45. Jenkins C, Moir S, Chan J, Rakhit D, Haluska B, Marwick TH. Left ventricular volume measurement with echocardiography: a comparison of left ventricular opacification, three-dimensional echocardiography, or both with magnetic resonance imaging. Eur. Heart J. 30(1), 98-106 (2009).

46. To AC, Dhillon A, Desai MY. Cardiac magnetic resonance in hypertrophic cardiomyopathy. JACC Cardiovasc. Imaging 4(10), 1123-1137 (2011). 
47. Puntmann VO, Jahnke C, Gebker $\mathrm{R}$ et al. Usefulness of magnetic resonance imaging to distinguish hypertensive and hypertrophic cardiomyopathy. Am. J. Cardiol. 106(7), 1016-1022 (2010).

48. Lang RM, Bierig M, Devereux RB et al. Recommendations for chamber quantification. Eur. J. Echocardiogr. 7(2), 79-108 (2006).

49. Maron MS, Maron BJ, Harrigan C et al. Hypertrophic cardiomyopathy phenotype revisited after 50 years with cardiovascular magnetic resonance. J. Am. Coll. Cardiol. 54(3), 220-228 (2009).

50. Bruder O, Wagner A, Jensen CJ et al. Myocardial scar visualized by cardiovascular magnetic resonance imaging predicts major adverse events in patients with hypertrophic cardiomyopathy. J. Am. Coll. Cardiol. 56(11), 875-887 (2010).

51. O'Hanlon R, Grasso A, Roughton M et al. Prognostic significance of myocardial fibrosis in hypertrophic cardiomyopathy. J. Am. Coll. Cardiol. 56(11), 867-874 (2010).

52. Ismail TF, Jabbour A, Gulati A et al. Role of late gadolinium enhancement cardiovascular magnetic resonance in the risk stratification of hypertrophic cardiomyopathy. Heart 100(23), 1851-1858 (2014).

53. Chan RH, Maron BJ, Olivotto I et al. Prognostic value of quantitative contrast-enhanced cardiovascular magnetic resonance for the evaluation of sudden death risk in patients with hypertrophic cardiomyopathy. Circulation 130(6), 484-495 (2014).

54. Nordin S, Dancy L, Moon JC, Sado DM. Clinical applications of multiparametric CMR in left ventricular hypertrophy. Int. J. Cardiovasc. Imaging 34(4), 577-585 (2018).

55. Weng Z, Yao J, Chan RH et al. Prognostic value of LGE-CMR in HCM: a meta-analysis. JACC Cardiovasc. Imaging 9(12), 1392-1402 (2016).

56. Freitas P, Ferreira AM, Arteaga-Fernandez E et al. The amount of late gadolinium enhancement outperforms current guideline-recommended criteria in the identification of patients with hypertrophic cardiomyopathy at risk of sudden cardiac death. $J$. Cardiovasc. Magn. Reson. 21(1), 50 (2019).

57. van Heerebeek L, Paulus WJ. Understanding heart failure with preserved ejection fraction: where are we today? Neth. Heart J. 24(4), 227-236 (2016).

58. Paulus WJ, Tschope C. A novel paradigm for heart failure with preserved ejection fraction: comorbidities drive myocardial dysfunction and remodeling through coronary microvascular endothelial inflammation. J. Am. Coll. Cardiol. 62(4), 263-271 (2013).

59. Puntmann VO, Peker E, Chandrashekhar Y, Nagel E. T1 mapping in characterizing myocardial disease: a comprehensive review. Circ. Res. 119(2), 277-299 (2016).

60. Rommel KP, Lucke C, Lurz P. Diagnostic and prognostic value of CMR T1-mapping in patients with heart failure and preserved ejection fraction. Rev. Esp. Cardiol. (Engl. Ed.) 70(10), 848-855 (2017).

61. Su MY, Lin LY, Tseng YH et al. CMR-verified diffuse myocardial fibrosis is associated with diastolic dysfunction in HFpEF. JACC Cardiovasc. Imaging 7(10), 991-997 (2014).

62. Duca F, Kammerlander AA, Zotter-Tufaro C et al. Interstitial fibrosis, functional status, and outcomes in heart failure with preserved ejection fraction: insights from a prospective cardiac magnetic resonance imaging study. Circ. Cardiovasc. Imaging 9(12), (2016).

63. Rommel KP, von Roeder M, Latuscynski $\mathrm{K}$ et al. Extracellular volume fraction for characterization of patients with heart failure and preserved ejection fraction. J. Am. Coll. Cardiol. 67(15), 1815-1825 (2016).

64. Hen Y, Takara A, Iguchi $\mathrm{N}$ et al. High signal intensity on T2-weighted cardiovascular magnetic resonance imaging predicts life-threatening arrhythmic events in hypertrophic cardiomyopathy patients. Circ J 82(4), 1062-1069 (2018).

65. Gommans DHF, Cramer GE, Bakker J et al. High T2-weighted signal intensity for risk prediction of sudden cardiac death in hypertrophic cardiomyopathy. Int. J. Cardiovasc. Imaging 34(1), 113-120 (2018).

66. Amano Y, Aita K, Yamada F, Kitamura M, Kumita S. Distribution and clinical significance of high signal intensity of the myocardium on T2-weighted images in 2 phenotypes of hypertrophic cardiomyopathy. J. Comput. Assist. Tomogr. 39(6), 951-955 (2015).

67. Aletras AH, Tilak GS, Natanzon A et al. Retrospective determination of the area at risk for reperfused acute myocardial infarction with T2-weighted cardiac magnetic resonance imaging: histopathological and displacement encoding with stimulated echoes (DENSE) functional validations. Circulation 113(15), 1865-1870 (2006).

68. Yin L, Xu HY, Zheng SS et al. 3.0 T magnetic resonance myocardial perfusion imaging for semi-quantitative evaluation of coronary microvascular dysfunction in hypertrophic cardiomyopathy. Int. J. Cardiovasc. Imaging 33(12), 1949-1959 (2017).

69. Chen S, Huang L, Zhang Q, Wang J, Chen Y. T2-weighted cardiac magnetic resonance image and myocardial biomarker in hypertrophic cardiomyopathy. Medicine (Baltimore) 99(23), e20134 (2020).

- Provides the basis for further investigation into the correlation of hyperintensity on T2 imaging of hypertrophic cardiomyopathy patients and biomarkers of myocardial injury in these same patients. 
